Aus der Abteilung für Zahnerhaltungskunde und Präventivzahnmedizin des CharitéCentrums 3 für Zahn-, Mund- und Kieferheilkunde der Medizinischen Fakultät Charité - Universitätsmedizin Berlin

\author{
DISSERTATION
}

\title{
Penetration of micro-filled infiltrant resins into artificial caries lesions
}

\author{
zur Erlangung des akademischen Grades \\ Doctor medicinae dentariae (Dr. med. dent.)
}

\begin{abstract}
vorgelegt der Medizinischen Fakultät
Charité - Universitätsmedizin Berlin
\end{abstract}

von

\section{Haitham Askar}

aus Giza, Ägypten

Datum der Promotion: 10.03.2017 


\section{Inhaltsverzeichnis}

1. Zusammenfassung 3

2. Abstract 4

3. Ausführliche Anteilserklärung / eidesstattliche Versicherung 5

4. Journal summary list 6

5. Ausgewählte Publikation $\quad 7$

6. Lebenslauf 14

7. Publikationsliste 16

8. Danksagung 17 


\section{Zusammenfassung}

Ziel: Infiltranten wurden ursprünglich für die Arretierung nicht-kavitierter Karies entwickelt. Ein Zusatz von Füllstoffen könnte eine Behandlung auch von mikro-kavitierten Läsionen ermöglichen, sofern hierdurch die Infiltration nicht signifikant beeinträchtigt würde. Das Ziel dieser Studie war, den Einfluss verschiedener Füllkörper [org. Füller mittlere Korngröße $83 \mu \mathrm{m}$ (OF83) bzw. $42 \mu \mathrm{m}$ (OF42), Glas-Füller mittlere Korngröße 0,7 $\mu \mathrm{m}$ (GF0,7)] sowie der Konzentration des Füllkörper auf die Penetration experimenteller mikrogefüllter Infiltranten in künstliche kariöse Läsionen zu evaluieren.

Material und Methode: Bovine Schmelzproben $(n=180)$ wurden 100 Tage in Demineralisationslösung gelagert, um je drei künstliche kariöse Läsionen zu schaffen. Die Proben wurden randomisiert auf 10 Gruppen verteilt ( $\mathrm{n}=18)$ : (1) I (Icon; DMG; Kontrolle), (2) IOF83 (65\% Icon + 35\% OF83), (3) I-OF83 (55\% Icon + 45\% OF83), (4) I-OF83 (45\% Icon + $55 \%$ OF83), (5) I-OF42 (65\% Icon + 35\% OF42), (6) I-OF42 (55\% Icon + 45\% OF42), (7) IOF42 (45\% Icon + 55\% OF42), (8) I-GF0,7 (65\% Icon + 35\% GF0,7), (9) I-GF0,7 (55\% Icon + 45\% GF0,7), (10) I-GF0,7 (45\% Icon + 55\% GF0,7). In jeder Probe wurden zwei Läsionen für 5 s geätzt $\left(37 \% \mathrm{H}_{3} \mathrm{PO}_{4}\right)$ und mit dem jeweiligen Kunststoff für $5 \mathrm{~s}$ bzw. $10 \mathrm{~s}$ infiltriert. Die dritte Läsion diente als unbehandelte Kontrolle. Die Analyse der Penetrations- und Läsionstiefe (PT und LT) sowie der prozentualen Penetration $(\mathrm{PP}=\mathrm{PT} \times 100 / \mathrm{LT})$ erfolgte mittels CLSM.

Ergebnisse: Für beiden Anwendungszeiten beeinflusste die Größe des Füllkörper signifikant den PP, aber nicht die Konzentration des Füllkörper ( $p<0.05$; two-way ANOVA). PP der IOF42 zeigte keinen signifikanten Unterschied von ungefüllten Infiltranten, jedoch war dieser erheblich höher als der von OF83 und GF0.7 ( $\mathrm{p}<0.05$; two-way ANOVA).

\section{Schlussfolgerung:}

Die mit organischen Füllern versetzten Infiltranten (I-OF42) zeigten ähnliche Penetrationseigenschaften in künstliche Schmelzkaries wie der ungefüllte Infiltrant.

Stichworte: Kariesinfiltration, Icon, Infiltrant, Füllkörper, Schmelzkaries, CLSM. 


\begin{abstract}
Objectives: Infiltrants are non-filled low-viscosity resins that have been developed to arrest lesion progression by penetrating into the porosities of non-cavitated lesions where they are hardened and arrest lesion progression. The addition of fillers to infiltrant resin might combine the high penetrativity of the former with the better applicability of composite resins. The aim of the present study was to evaluate the penetration of different experimental micro-filled infiltrant resins (MFIRs) into artificial enamel lesions.
\end{abstract}

Methods: An infiltrant (Icon; DMG) was mixed with either one of three fillers [OF83: organic filler (83 mm), OF42: organic filler $(42 \mathrm{~mm})$ or GF0.7: glass filler $(0.7 \mathrm{~mm})]$ reaching 35, 45 and $55 \%$ filler content, respectively. In each of 180 bovine enamel specimens three artificial lesions were created; two lesions were etched for $5 \mathrm{~s}\left(37 \% \mathrm{H}_{3} \mathrm{PO}_{4}\right)$, leaving one lesion as control. Specimens were randomly allocated to 10 groups, in which either one of the MFIRs or the infiltrant was applied onto the two etched lesions for either $5 \mathrm{~s}$ or $10 \mathrm{~s}(\mathrm{n}=18 /$ group). Percentage of penetration (PP) was calculated and analysed.

Results: For both application times a significant influence of filler size but not of filler concentration on PP could be revealed ( $\mathrm{p}<0.05$; two-way ANOVA). PP of MFIRs-OF42 was not significantly different from unfilled infiltrant but significantly higher than those of OF83 and GF0.7 ( $<<0.05$; two-way ANOVA).

Conclusions: MFIRs-OF42 showed the ability to penetrate into artificial enamel caries lesions similar to the unfilled infiltrant resin.

Clinical significance: MFIRs could provide a new micro-invasive treatment for small-cavitated lesions.

Key words: Caries infiltration, Icon, Infiltrant, fillers, enamel caries, CLSM. 


\section{Eidesstattliche Versicherung}

„Ich, Haitham Askar, versichere an Eides statt durch meine eigenhändige Unterschrift, dass ich die vorgelegte Dissertation mit dem Thema: Penetration of micro-filled infiltrant resins into artificial caries lesions selbstständig und ohne nicht offengelegte Hilfe Dritter verfasst und keine anderen als die angegebenen Quellen und Hilfsmittel genutzt habe.

Alle Stellen, die wörtlich oder dem Sinne nach auf Publikationen oder Vorträgen anderer Autoren beruhen, sind als solche in korrekter Zitierung (siehe „Uniform Requirements for Manuscripts (URM)“ des ICMJE -www.icmje.org) kenntlich gemacht. Die Abschnitte zu Methodik (insbesondere praktische Arbeiten, Laborbestimmungen, statistische Aufarbeitung) und Resultaten (insbesondere Abbildungen, Graphiken und Tabellen) entsprechen den URM (s.o) und werden von mir verantwortet.

Mein Anteil an der ausgewählten Publikation entspricht dem, der in der untenstehenden gemeinsamen Erklärung mit dem/der Betreuer/in, angegeben ist.

Die Bedeutung dieser eidesstattlichen Versicherung und die strafrechtlichen Folgen einer unwahren eidesstattlichen Versicherung (§156,161 des Strafgesetzbuches) sind mir bekannt und bewusst.“"

Datum

Unterschrift

\section{$\underline{\text { Ausführliche Anteilserklärung an der erfolgten Publikation }}$}

Askar H, Lausch J, Dörfer CE, Meyer-Lueckel H, Paris S. Penetration of micro-filled infiltrant resins into artificial caries lesions. J Dent 2015;43(7):832-8.

Beitrag im Einzelnen (bitte ausführlich ausführen):

- H.A. entwickelte und führte die Studie durch, analysierte sowie interpretierte die gewonnenen Daten und schrieb das Manuskript zu entsprechender Studie.

- J.L. analysierte, interpretierte die Daten und überprüfte das Manuskript.

- C.D. analysierte, interpretierte die Daten und überprüfte das Manuskript.

- H.ML. entwickelte die Studie, analysierte, interpretierte die Daten und überprüfte das Manuskript.

- S.P. entwickelte die Studie, analysierte, interpretierte die Daten und überprüfte das Manuskript.

Unterschrift, Datum und Stempel des betreuenden Hochschullehrers/der betreuenden Hochschullehrerin

Unterschrift des Doktoranden/der Doktorandin 
ISI Web of Knowledge

Journal Citation Reports ${ }^{\circledast}$

I- WELCOME ? HELP

2015 JCR Science Edition

Journal Summary List

$\underline{\text { Journal Title Changes }}$

Journals from: subject categories DENTISTRY, ORAL SURGERY \& MEDICINE VI VEW CATEGORY SUMMARY LIT

Sorted by: Impact Factor $\quad$ SORT AGAIN

Journals 1 - 20 (of 89)

MARK ALL UPDATE MARKED LIST

$|\langle\langle[1|\underline{2}| \underline{3}|\underline{4}| \underline{5}]\rangle\rangle|$

Page 1 of 5

Ranking is based on your journal and sort selections.

\begin{tabular}{|c|c|c|c|c|c|c|c|c|c|c|c|}
\hline \multirow[b]{2}{*}{ Mark } & \multirow[b]{2}{*}{ Rank } & \multirow[b]{2}{*}{$\begin{array}{c}\text { Abbreviated Journal Title } \\
\text { (linked to journal } \\
\text { information) }\end{array}$} & \multirow[b]{2}{*}{ ISSN } & \multicolumn{6}{|c|}{ JCR Data i } & \multicolumn{2}{|c|}{ Eigenfactor ${ }^{\circledR}$ Metrics i } \\
\hline & & & & $\begin{array}{l}\text { Total } \\
\text { Cites }\end{array}$ & $\begin{array}{l}\text { Impact } \\
\text { Factor }\end{array}$ & $\begin{array}{l}\text { 5-Year } \\
\text { Impact } \\
\text { Factor }\end{array}$ & $\underset{\text { Index }}{\text { Immediacy }}$ & Articles & $\begin{array}{c}\text { Cited } \\
\text { Half- } \\
\text { life }\end{array}$ & $\begin{array}{l}\left.\text { Eigenfactor }^{(}\right) \\
\text {Score }\end{array}$ & $\begin{array}{c}\text { Article } \\
\text { Influence }^{\circledR} \\
\text { Score }\end{array}$ \\
\hline$\square$ & 1 & PERIODONTOL 2000 & $\begin{array}{l}0906- \\
6713\end{array}$ & 3376 & 4.949 & 5.108 & 1.311 & 45 & 9.7 & 0.00442 & 1.504 \\
\hline$\square$ & 2 & $\underline{\text { J DENT RES }}$ & $\begin{array}{l}0022- \\
0345\end{array}$ & 16401 & 4.602 & 5.024 & 0.837 & 233 & 9.9 & 0.02331 & 1.530 \\
\hline$\square$ & 3 & ORAL ONCOL & $\begin{array}{l}1368- \\
8375\end{array}$ & 7291 & 4.286 & 3.752 & 0.969 & 159 & 6.2 & 0.01494 & 1.132 \\
\hline$\square$ & 4 & CLIN IMPLANT DENT R & $\begin{array}{l}1523- \\
0899\end{array}$ & 3161 & 4.152 & 4.500 & 0.452 & 199 & 4.6 & 0.00757 & 1.275 \\
\hline$\square$ & 5 & DENT MATER & $\begin{array}{l}0109- \\
5641\end{array}$ & 10324 & 3.931 & 4.667 & 0.674 & 187 & 7.2 & 0.01323 & 1.007 \\
\hline$\square$ & 6 & $\underline{\text { J CLIN PERIODONTOL }}$ & $\begin{array}{l}0303- \\
6979\end{array}$ & 11767 & 3.915 & 4.435 & 0.658 & 155 & 9.5 & 0.01380 & 1.225 \\
\hline$\square$ & 7 & CLIN ORAL IMPLAN RES & $\begin{array}{l}0905- \\
7161\end{array}$ & 11968 & 3.464 & 4.522 & 0.619 & 236 & 7.2 & 0.01661 & 1.095 \\
\hline$\nabla$ & 8 & J DENT & $\begin{array}{l}0300- \\
5712\end{array}$ & 6199 & 3.109 & 3.254 & 0.543 & 184 & 6.7 & 0.01102 & 0.875 \\
\hline$\square$ & 9 & MOL ORAL MICROBIOL & $\begin{array}{l}2041- \\
1006\end{array}$ & 577 & 3.061 & 3.092 & 1.028 & 36 & 3.3 & 0.00262 & 0.992 \\
\hline$\square$ & 10 & $\underline{\text { J ENDODONT }}$ & $\begin{array}{l}0099- \\
2399\end{array}$ & 12519 & 2.904 & 3.357 & 0.394 & 335 & 6.7 & 0.01616 & 0.662 \\
\hline$\square$ & 11 & $\underline{\text { J PERIODONTOL }}$ & $\begin{array}{l}0022- \\
3492\end{array}$ & 14268 & 2.844 & 3.113 & 0.727 & 161 & $>10.0$ & 0.01306 & 0.839 \\
\hline$\square$ & 12 & $\underline{\text { INT ENDOD J }}$ & $\begin{array}{l}0143- \\
2885\end{array}$ & 5253 & 2.842 & 2.794 & 0.928 & 139 & 7.7 & 0.00876 & 0.853 \\
\hline$\square$ & 13 & J OROFAC PAIN & $\begin{array}{l}1064- \\
6655\end{array}$ & 1280 & 2.824 & 2.678 & & 0 & 9.9 & 0.00194 & 0.896 \\
\hline$\square$ & 14 & OPER DENT & $\begin{array}{l}0361- \\
7734\end{array}$ & 2593 & 2.819 & 2.207 & 0.537 & 95 & 7.9 & 0.00336 & 0.560 \\
\hline$\square$ & 15 & $\underline{\text { INT J ORAL SCI }}$ & $\begin{array}{l}1674- \\
2818\end{array}$ & 678 & 2.595 & 3.203 & 0.406 & 32 & 3.8 & 0.00251 & 0.936 \\
\hline$\square$ & 16 & $\underline{\text { J PERIODONTAL RES }}$ & $\begin{array}{l}0022- \\
3484\end{array}$ & 3662 & 2.474 & 2.394 & 0.622 & 98 & 9.1 & 0.00493 & 0.661 \\
\hline$\square$ & 17 & $\underline{\text { J ORAL FACIAL PAIN H }}$ & $\begin{array}{l}2333- \\
0384\end{array}$ & 102 & 2.444 & 2.444 & 0.359 & 39 & 1.4 & 0.00040 & 0.741 \\
\hline$\square$ & 18 & EUR J ORAL IMPLANTOL & $\begin{array}{l}1756- \\
2406\end{array}$ & 648 & 2.328 & 3.736 & 0.214 & 28 & 4.4 & 0.00239 & 1.128 \\
\hline$\square$ & 19 & CARIES RES & $\begin{array}{l}0008- \\
6568\end{array}$ & 3746 & 2.278 & 3.174 & 0.397 & 78 & $>10.0$ & 0.00454 & 0.871 \\
\hline$\square$ & 20 & COMMUNITY DENT ORAL & $\begin{array}{l}0301- \\
5661\end{array}$ & 3686 & 2.233 & 2.357 & 0.311 & 61 & $>10.0$ & 0.00405 & 0.763 \\
\hline
\end{tabular}


Askar, H., et al., Penetration of micro-filled infiltrant resins into artificial caries lesions. J Dent, 2015. 43(7): p. 832-8.

http://dx.doi.org/10.1016/j.jdent.2015.03.002 


\section{Lebenslauf}

Mein Lebenslauf wird aus datenschutzrechtlichen Gründen in der elektronischen Version meiner Arbeit nicht veröffentlicht. 


\section{Lebenslauf}

Mein Lebenslauf wird aus datenschutzrechtlichen Gründen in der elektronischen Version meiner Arbeit nicht veröffentlicht. 


\section{Publikationsliste:}

1. Askar H, Lausch J, Dorfer CE, Meyer-Lueckel H, Paris S. Penetration of micro-filled infiltrant resins into artificial caries lesions. J Dent 2015;43(7):832-8. (Impact factor: 3,109 )

2. Brouwer F, Askar H, Paris S, Schwendicke F. Detecting Secondary Caries Lesions: A Systematic Review and Meta-analysis. J Dent Res 2015;95(2):143-51. (Impact factor: $4,602)$

3. Umwali A, Askar H, Paris S, Schwendicke F. Radiographic, antibacterial and bondstrength effects of radiopaque caries tagging. Sci Rep 2016;6:27319. (Impact factor: $5,228)$

\section{Kongresse:}

1. H. Askar, J. Lausch, C.E. Dörfer, H. Meyer-Lueckel, S. Paris. Penetration mikrogefüllter Infiltranten in künstliche Kariesläsionen 2. Gemeinschaftstagung der DGZ und der DGET mit der DGPZM und der DGR²Z, Munich, Germany, October 2015

2. H. Askar, J. Lausch, H. Meyer-Lueckel, S. Paris. Effect of surface pre-treatment on resin infiltration of active and in-active natural enamel caries lesions, $62^{\text {nd }}$ Congress of the European Organisation for Caries Research (ORCA), Brussels, Belgium, July 2015

3. H. Askar, J. Lausch, H. Meyer-Lueckel, S. Paris, Modified resin infiltration of natural caries lesions varying in ICDAS-codes in vitro. $61^{\text {st }}$ Congress of the European Organisation for Caries Research (ORCA), Greifswald, Germany, July 2014

4. H. Askar, H. Meyer-Lueckel, J.G. Noren, C.E. Dörfer, S. Paris, Influence of Sodium Hypochlorite Pre-Treatment on Resin Infiltration of MI-Hypomineralized Enamel. $60^{\text {th }}$ Congress of the European Organisation for Caries Research (ORCA), Liverpool, UK, July 2013

5. S. Paris, H. Askar, C.E. Dorfer, J.G. Noren, H. Meyer-Lueckel. Resin infiltration of Hypomineralized Enamel in 'MIH-Molars'. IADR/AADR/CADR General Session and Exhibition, Seattle, USA, March 2013 


\section{Danksagung}

Ohne den Rat und die Unterstützung anderer wäre die vorliegende Dissertation nicht möglich gewesen. Deshalb möchte ich jenen Personen meinen aufrichtigen Dank aussprechen, die mich im Rahmen dieser wissenschaftlichen Arbeit unterstützt haben.

Mein größter Dank gebührt Herrn Prof. Dr. Sebastian Paris (Abteilung für Zahnerhaltung und Präventivzahnmedizin, Charité-Universitätsmedizin Berlin), der mir jederzeit für konstruktive Gespräche zur Verfügung stand und mir über jede Hürde fachlich und menschlich hinweggeholfen hat. Er hat mir vielfältige Möglichkeiten eröffnet, mich wissenschaftlich in der Abteilung für Zahnerhaltung und Präventivzahnmedizin der Charité zu entwickeln.

Mein besonderer Dank gilt Herrn Prof. Dr. Hendrik Meyer-Lückel (Klinik für Zahnerhaltungskunde, Parodontologie und Präventive Zahnheilkunde der RWTH Aachen), der mir die Möglichkeit gegeben hat, diese Arbeit durchzuführen und der mich fachlich betreut und unterstützt hat.

Mein Dank geht an das gesamte Kollegium der Abteilung für Zahnerhaltung und Präventivzahnmedizin der Charité, hier im Besonderen Herrn OA Priv.-Doz. Dr. Falk Schwendicke für die produktive wissenschaftliche Zusammenarbeit.

Herrn Prof. Dr. C. Dörfer (Klinik für Zahnerhaltungskunde und Parodontologie, ChristianAlbrechts-Universität zu Kiel) danke ich ebenso wie der dortigen gesamten Arbeitsgruppe im Forschungslabor. Für die professionelle und immer freundliche Einführung und fortwährende Hilfe in sämtliche labortechnische Arbeiten danke ich Herrn Michael Stiebritz (Klinik für Zahnerhaltungskunde, Parodontologie und Präventive Zahnheilkunde der RWTH Aachen).

Bei Herrn Prof. Dr. Mohamed Zaazou (Abteilung für Zahnerhaltung, Nationales Forschungszentrum, Kairo, Ägypten) und Herrn Prof. Dr. Nour Habib (Abteilung für Biomaterial, Universität Kairo, Ägypten) möchte ich mich für die Unterstützung bedanken, die ein wichtiger Beitrag zum Gelingen meines Werdegangs war.

Bedanken möchte ich mich auch beim Ministerium für Hochschulbildung in Kairo, die in enger Zusammenarbeit mit dem DAAD (Deutschen Akademischen Austauschdienst, Kairo) stehen und mir ein Stipendium ermöglicht haben, das mir den Weg in die Zahnmedizin nach Deutschland geebnet hat.

Ein ganz besonderer Dank geht an Frau Angelika Cernitori und Frau Pamela Glowacki (Charité International Cooperation), die mich bei allen administrativen Abläufen an der Charité und an jeglicher Behörde durch stetige Hilfe begleitet haben und mir darüber hinaus jeden anderen wertvollen, unterstützenden Beistand geben konnten.

Zuletzt möchte ich mich bei meiner Familie, insbesondere bei meinen Eltern und meinem Bruder, bedanken. Sie waren immer bereit, das Beste für mich zu tun und alles Erdenkliche dafür zu geben. Sie haben mich immer motiviert und in jeder Weise bestärkt. Danke auch meinen Freunden, die mir in allen Phasen meines Lebens zur Seite gestanden haben. 